\title{
Using mRNA and small RNA gene expression changes in peripheral blood for easy detection of Ra-223 incorporation
}

\author{
Patrick Ostheim ${ }^{1, *}$, Matthäus Majewski ${ }^{1}$, Matthias Miederer $^{2}$, Mathias Schreckenberger ${ }^{2}$, \\ Tim Nestler ${ }^{3}$, Manuela A. Hoffmann ${ }^{4}$, Matthias Port ${ }^{1}$, and Michael Abend ${ }^{1}$ \\ ${ }^{1}$ Bundeswehr Institute of Radiobiology, Munich, Germany \\ ${ }^{2}$ University Medical Center of the Johannes Gutenberg University, Clinic and Polyclinic for Nuclear \\ Medicine, Mainz, Germany \\ ${ }^{3}$ Department of Urology, Federal Armed Services Hospital Koblenz, Koblenz, Germany \\ ${ }^{4}$ Supervisory Center for Medical Radiation Protection, Bundeswehr Medical Service Headquarters, \\ Koblenz, Germany
}

\section{Background}

Radiation analytics is the established method for detection of incorporated alpha-emitting radionuclides. It is laborious and time consuming. We wondered whether changes in gene expression measured in the human peripheral blood after radionuclide incorporation might serve as an easier approach for detection of incorporated radionuclides in a radiological or nuclear scenario.

\section{Methods and Results}

We received whole blood in PAXgene Blood RNA tubes from four patients suffering from multiple bone-metastatic, castration-resistant prostate cancer (without visceral or nodal involvement), who underwent treatment with the alpha emitting isotope radium-223 dichloride (Ra-223, Xofigo $\left.{ }^{\circledR}\right)$. Patients received about $4 \mathrm{MBq}$ per cycle and month and were treated for six months.

In a first step, we employed next generation sequencing (NGS) for a whole genome screening of the transcriptome (mRNAs) as well as the post-transcriptome including small RNAs (e.g. long non-coding RNAs, snoRNAs and miRNAs) in one patient at 8 different time points during 6 cycles of Ra-223-therapy. This allowed us to identify specific candidate RNAs which are associated with the increased dose cumulated over the treatment time. 
In a second step, candidate mRNAs and small RNAs which appeared dose-dependently differentially up- or down-regulated $(>2$-fold, $\mathrm{p}<0.05)$ in the screening samples will be chosen for validation. This will be performed by using the remaining patient samples $(n=3$ patients, each with up to 4 points in time) and shifting to more sensitive and specific qRTPCR platform at the same time.

\section{Conclusion/Prospect}

We will present and discuss the results on radiation-induced gene expression changes in whole blood which might serve as a simplified diagnostic tool for identification of incorporated radionuclides (Ra-223) in future.

\section{Key words}

Radiation analysis, incorporation, gene expression changes, radiation biomarkers, small RNA, mRNA, transcriptome, prostate cancer, Radium-223. 